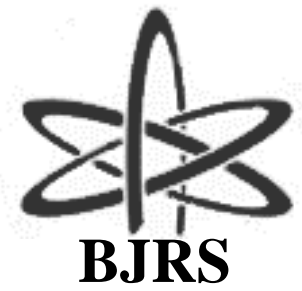

\author{
BRAZILIAN JOURNAL \\ $\mathrm{OF}$ \\ RADIATION SCIENCES \\ 07-03A (2019) 01-13
}

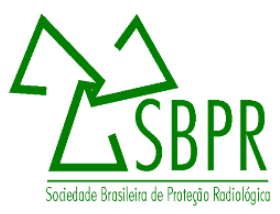

\title{
Avaliação dos efeitos da irradiação gama no composto gel clínico/sulfato de cobre analisados em imagens de
} ressonância magnética

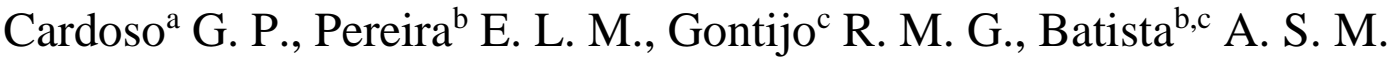 \\ ${ }^{a}$ Centro de Desenvolvimento da Tecnologia Nuclear (CDTN), 31270-901, Avenida Presidente Antônio Carlos, 6627, \\ Pampulha, Belo Horizonte, Minas Geras, Brasil. \\ gabrielapontesc@gmail.com \\ ${ }^{b}$ Departamento de Engenharia Nuclear, Universidade Federal de Minas Gerais (UFMG), 31270-901, Avenida \\ Presidente Antônio Carlos, 6627, Pampulha, Belo Horizonte, Minas Geras, Brasil. \\ esther_machado@outlook.com.br \\ ${ }^{c}$ Departamento de Anatomia e Imagem, Universidade Federal de Minas Gerais (UFMG), 30130-100, Avenida \\ Professor Alfredo Balena, 190, Santa Efigênia, Belo Horizonte, Minas Gerais, Brasil.
}

adriananuclear@yahoo.com.br

\section{RESUMO}

Simuladores de corpo para Ressonância Magnética (RM) são úteis para treinamento de novos profissionais e também no controle do bom funcionamento dos equipamentos. Este trabalho trata-se de uma avaliação de composto de gel clínico incolor com sulfato de cobre penta-hidratado $\left(\mathrm{CuSO}_{4} .5 \mathrm{H}_{2} \mathrm{O}\right)$, em diferentes proporções, para o uso como simulador de corpo em exames de RM. As amostras foram irradiadas com doses gama de 10, 50 e $100 \mathrm{kGy}$. Imagens de RM foram obtidas em um equipamento GE, 1.5 Tesla, do Hospital das Clínicas da Universidade Federal de Minas Gerais (HC-UFMG) e analisadas com o software ImageJ®, demonstrando diferenças nos tempos de relaxação promovidos pelo processo de irradiação. Amostras de gel puro e de baixas concentrações de sulfato de cobre apresentaram bom contraste nas imagens de RM, relacionado com o processo de irradiação. $\mathrm{O}$ mesmo ocorre em concentrações maiores de $\mathrm{CuSO}_{4} .5 \mathrm{H}_{2} \mathrm{O}$ não irradiadas.

Palavras-chave: Simulador de Corpo, Ressonância Magnética, Irradiação Gama, Gel Clínico, CuSO $\mathrm{O}_{4}$ 


\begin{abstract}
Magnetic Resonance Image (MRI) body simulators are useful for training new professionals and also for controlling the proper functioning of the equipment. This work is an evaluation of colorless clinical gel compound with copper sulfate pentahydrate $\left(\mathrm{CuSO}_{4} .5 \mathrm{H}_{2} \mathrm{O}\right)$, in different proportions, for use as a body simulator in MR examinations. Samples were irradiated with gamma doses of 10, 50 and $100 \mathrm{kGy}$. MR images were obtained in a GE equipment, 1.5 Tesla, of the Hospital das Clínicas of the Universidade Federal de Minas Gerais (HC-UFMG) and analyzed with Image ${ }^{\circledR}$ software, demonstrating differences in the relaxation times promoted by the irradiation process. Samples of pure gel and low concentrations of copper sulfate presented good contrast in the MRI images, related to the irradiation process. The same occurs at higher concentrations of unirradiated $\mathrm{CuSO}_{4} .5 \mathrm{H}_{2} \mathrm{O}$.
\end{abstract}




\section{INTRODUÇÃO}

A ressonância magnética $(\mathrm{RM})$ é uma técnica de diagnóstico por imagem bastante atrativa por possibilitar a visibilidade de órgãos e tecidos moles do corpo humano sem necessidade do uso de radiação ionizante. Seu desenvolvimento é recente em relação a outras técnicas por imagem resultando em equipamentos caros e com manutenção onerosa [1]. Neste sentido o treinamento de profissionais para manuseio da técnica e obtenção de imagens diagnósticas de qualidade passa por um preparo teórico e necessariamente um manuseio prático. Devido à preocupação adicional da manipulação do equipamento por profissional em treinamento se faz necessário recursos didáticos, tais como simuladores de corpo, para concretizar o entendimento teórico dos parâmetros a serem trabalhados para obtenção da imagem.

Embora as imagens médicas tenham em comum o contraste baseado em diferença de tons de cinza, outras técnicas de diagnóstico por imagem, como Raios X (RX) e Tomografia Computadorizada (TC) possuem parâmetros de obtenção de imagem que se relacionam, tornando-as mais intuitivas, quanto às propriedades de atenuação dos tecidos [2]. Por outro lado, para aquisição de imagem por RM é necessária à compreensão de princípios físicos complexos e pouco intuitivos [3]. Os tons de cinza que determinam o contraste da imagem estão relacionados à manipulação de características intrínsecas dos tecidos, como os tempos de relaxação T1 e T2, densidade de prótons, a manipulação de parâmetros extrínsecos, como Tempo de Repetição (TR), Tempo de Eco (TE), Tempo de Inversão (TI), entre os principais [4]. É de valor na formação do futuro profissional a oportunidade de validar os conhecimentos teóricos, porém o acesso a RM é limitada pelo seu alto custo e, neste sentido, o manuseio do equipamento por alunos em formação deve ser em condições seguras e ainda, em simulação de exames, em função da presença de pacientes ou colaboradores voluntários adicionar gastos devido a necessidade da presença de um médico responsável.

Dentro deste contexto, o presente trabalho apresenta parte de um projeto de pesquisa e ensino de conceber um simulador de corpo para uso em RM que seja seguro, de baixo custo e fácil manipulação. Para tanto, é necessário material que, ao ser submetido ao campo magnético, retorne sinais de relaxação distintos, proporcionando variados tons de cinza. Estes seriam então correlacionados a diferentes tempos de relaxação T1 e T2 conforme a manipulação dos parâmetros 
extrínsecos. Para tanto, foi escolhido como material simulador o gel clínico incolor puro e com adição de sulfato de cobre penta-hidratado $\left(\mathrm{CuSO}_{4} .5 \mathrm{H}_{2} \mathrm{O}\right)$ em diferentes proporções. Adicionalmente foi testada a influência da exposição do gel e dos compostos a irradiação com radiação gama proveniente de uma fonte de cobalto $-60\left({ }^{60} \mathrm{Co}\right)$. O gel foi escolhido por ser composto essencialmente por água e carbono [5]. O hidrogênio presente na solução aquosa é responsável por produzir as imagens, o sulfato de cobre por modificar a resposta do hidrogênio [6] e a interação da radiação com o gel e composto previsto por modificar a configuração química do hidrogênio, eventualmente proporcionando diferença no sinal captado [7]. A comprovação da contribuição do processo de irradiação na obtenção de distintos tons de cinza passa pela obtenção da imagem do simulador em equipamento de RM e análise da mesma, sendo o objetivo do presente trabalho.

\section{MATERIAIS E MÉTODOS}

Para cada amostra foi utilizado $100 \mathrm{ml}$ de RMC® Gel Clínico incolor do fabricante Unigel puro e diferentes proporções de $\mathrm{CuSO}_{4} .5 \mathrm{H}_{2} \mathrm{O}$, os quais foram misturados manualmente formando sete amostras a serem analisadas: gel clínico puro, com 50, 100, 200, 300, 400 e $500 \mathrm{mg}$ de $\mathrm{CuSO}_{4} .5 \mathrm{H}_{2} \mathrm{O}$ dispersos. A homogeinedade conseguida através da mistura manual foi testada previamente utilizando Espectrometria no Ultravioleta Visível (UV-Vis) [8]. Após a mistura, uma fração de $3 \mathrm{ml}$ de cada amostra foi separada para irradiação no Laboratório de Irradiação Gama (LIG) do Centro de Desenvolvimento da Tecnologia Nuclear (CDTN) com doses de 10, 50 e 100 kGy em uma taxa de dose constante. Todas as amostras foram irradiadas com 10 e $50 \mathrm{kGy}$, amostras de gel puro foram irradiadas nas três doses (10, 50 e $100 \mathrm{kGy})$. Para efeito comparativo as amostras não irradiadas de gel puro e dos compostos também foram utilizadas na obtenção da imagem por RM, além de um recipiente vazio (para verificar sua influência na obtenção da imagem).

As amostras irradiadas foram colocadas juntas às não irradiadas em uma caixa de polipropileno e as imagens foram obtidas em um equipamento General Electric Company (GE) de 1.5 Tesla, do Hospital das Clínicas da Universidade Federal de Minas Gerais (HC-UFMG) e analisadas com o software Image ${ }^{\circledR}$ para verificar os tons de cinza resultantes na imagem. 
Foram utilizadas as sequências Fast Spin Eco (FSE) para obtenção das imagens ponderadas em T1 e sequências Fast Spin Eco com Restauração Magnética (FRFSE) para obtenção das imagens ponderadas em T2, com cortes axiais, coronais e sagitais. Para as imagens em FSE T1 foi utilizado como parâmetro para os cortes axiais TR de $850 \mathrm{~ms}$, TE de $8,7 \mathrm{~ms}$; para cortes coronais TR de 383,3 ms e TE de 8,7 ms; para cortes sagitais TR de 566,7 ms e TE de 8,7 ms. Para as imagens em FRFSE foram utilizados como parâmetros para cortes axiais TR de 9.850 ms e TE de 114,8 ms; para cortes coronais TR de 4.483,3 ms e TE de 115,6 ms; para cortes sagitais TR de 6.216,7 ms e TE de 115,6 ms. Foi utilizada bobina pediátrica para região do tórax na obtenção das imagens por se adequar melhor ao tamanho do simulador.

\section{RESULTADOS E DISCUSSÃO}

A imagem de RM é obtida com aplicação de um campo magnético que exerce uma força tão intensa que alinha os spins dos prótons de hidrogênio com orientação espacial definida, paralela e antiparalela. Este fenômeno confere o comportamento de mini-ímãs. A direção de aplicação do campo magnético é chamada de eixo $z$ ou longitudinal e o plano $x y$ é chamado de plano transversal. Para orientar os spins, emite-se um pulso de radiofrequência (RF) de curta duração, que deve estar em fase com a frequência de precessão [4]. O efeito produzido é o de desviar os spins do eixo longitudinal em um ângulo definido. O mais usado é o de $90^{\circ}$ que os alinha ao plano transversal, como os utilizados neste trabalho.

Quando se encerra a aplicação dos pulsos de RF, ocorre a relaxação dos spins que irão liberar a energia absorvida por esta ação e voltarão à orientação de acordo com o campo. As bobinas, que são acessórios específicos para cada finalidade e região do corpo, são usadas para emitir e captar estes sinais, fazendo a leitura da fase e da frequência para definir a localização de onde vem o sinal. O tempo do retorno correspondente a $63 \%$ do valor para a magnetização longitudinal é chamado T1. O tempo que a magnetização no plano transversal leva para atingir $37 \%$ do seu valor inicial é chamado T2 [4]. Na Fig. 1 apresentamos as imagens obtidas com a sequência FSE, ponderação em T1 e corte sagital. As amostras foram identificadas através de códigos a fim de possibilitar um comparativo com as imagens obtidas em coronal, sagital e na ponderação FRFSE em T2. 
Figura 1: Imagens obtidas das amostras em ponderação T1 sequência FSE e a codificação das amostras conforme composição e dose de radiação recebida.

\section{Sagital T1 FSE}

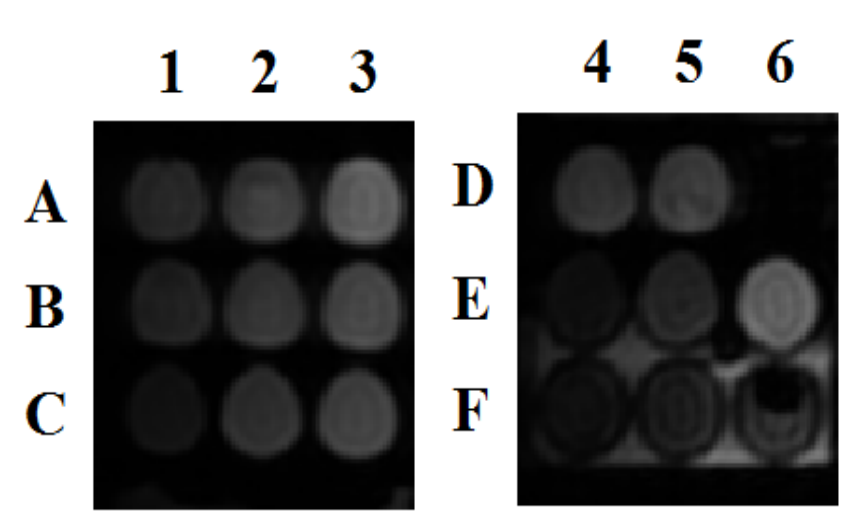

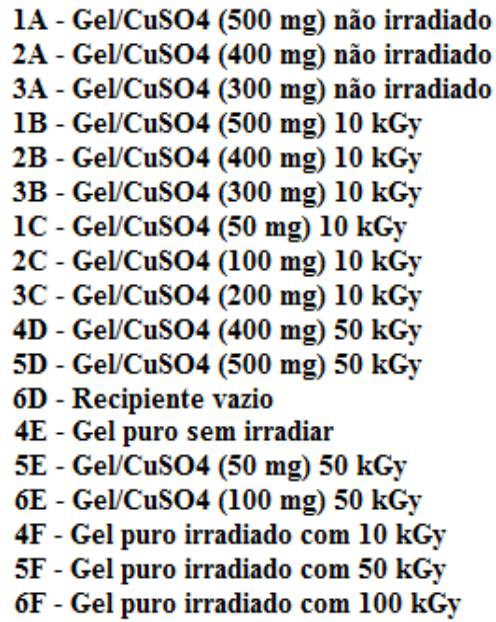

$1 \mathrm{~A}$ - Gel/CuSO4 (500 mg) não irradiado

2A - Gel/CuSO4 (400 mg) não irradiado

$1 \mathrm{~B}$ - Gel/CuSO4 (500 mg) $10 \mathrm{kGy}$

2B - Gel/CuSO4 (400 mg) $10 \mathrm{kGy}$

(

$2 \mathrm{C}$ - Gel/CuSO4 (100 mg) $10 \mathrm{kGy}$

3C - Gel/CuSO4 (200 mg) $10 \mathrm{kGy}$

4D - Gel/CuSO4 (400 mg) $50 \mathrm{kGy}$

$5 \mathrm{D}$ - Gel/CuSO4 (500 mg) $50 \mathrm{kGy}$

- Recipiente vazio

E - Gel puro sem irradiar

$4 \mathrm{~F}$ - Gel puro irradiado com $10 \mathrm{kG}$

$5 \mathrm{~F}$ - Gel puro irradiado com $50 \mathrm{kGy}$

$6 \mathrm{~F}$ - Gel puro irradiado com $100 \mathrm{kGy}$

Observamos na Fig. 1 que a imagem codificada 6D trata-se do recipiente vazio e que ele não contribui com a imagem, aparecendo somente como espaço escuro. Percebemos diferença nas tonalidades das demais amostras sendo algumas bem mais brilhantes que outras, demonstrando diferença na relaxação T1 entre elas. A Fig. 2 mostra imagens obtidas nos demais cortes, coronal e axial, para um comparativo em termos de brilho e diferença de tons de cinza, resultando em diferentes contrastes e a visibilidade do contorno dos recipientes em que as amostras foram colocadas. Pode-se observar pela posição em que foram colocadas uma formação de bolha no interior do recipiente. O programa Image ${ }^{\circledR}$ foi utilizado para estimar com precisão a diferença de tons de cinza conseguidos nas diferentes amostras.

$\mathrm{Na}$ Tab. 1 apresentamos as tonalidades de cinza calculadas pelo programa ImageJ® para as amostras de compostos gel/CuSO $4.5 \mathrm{H}_{2} \mathrm{O}$ nas proporções de 50, 100, 300, 400 e $500 \mathrm{mg}$ irradiadas com doses de 10 e $50 \mathrm{kGy}$. As tonalidades quantificadas foram obtidas através de histogramas das imagens originais em formato Digital Imaging and Communication in Medicine (DICOM) com valores que variam entre 0 a 150, sendo que os próximos de zero são mais escuros, tendendo ao preto e os próximos de 150 mais claros, tendendo ao branco. Ou seja, se maioria dos pixels estão 
mais próximos de zero significa uma imagem mais escura; ao contrário se a maioria dos pixels encontram-se mais próximos dos últimos valores do nível de cinza, então a imagem é mais brilhante [9].

Figura 2: Imagem das amostras obtidas em ponderação T1 sequência FSE codificadas nos cortes sagital, coronal e axial.

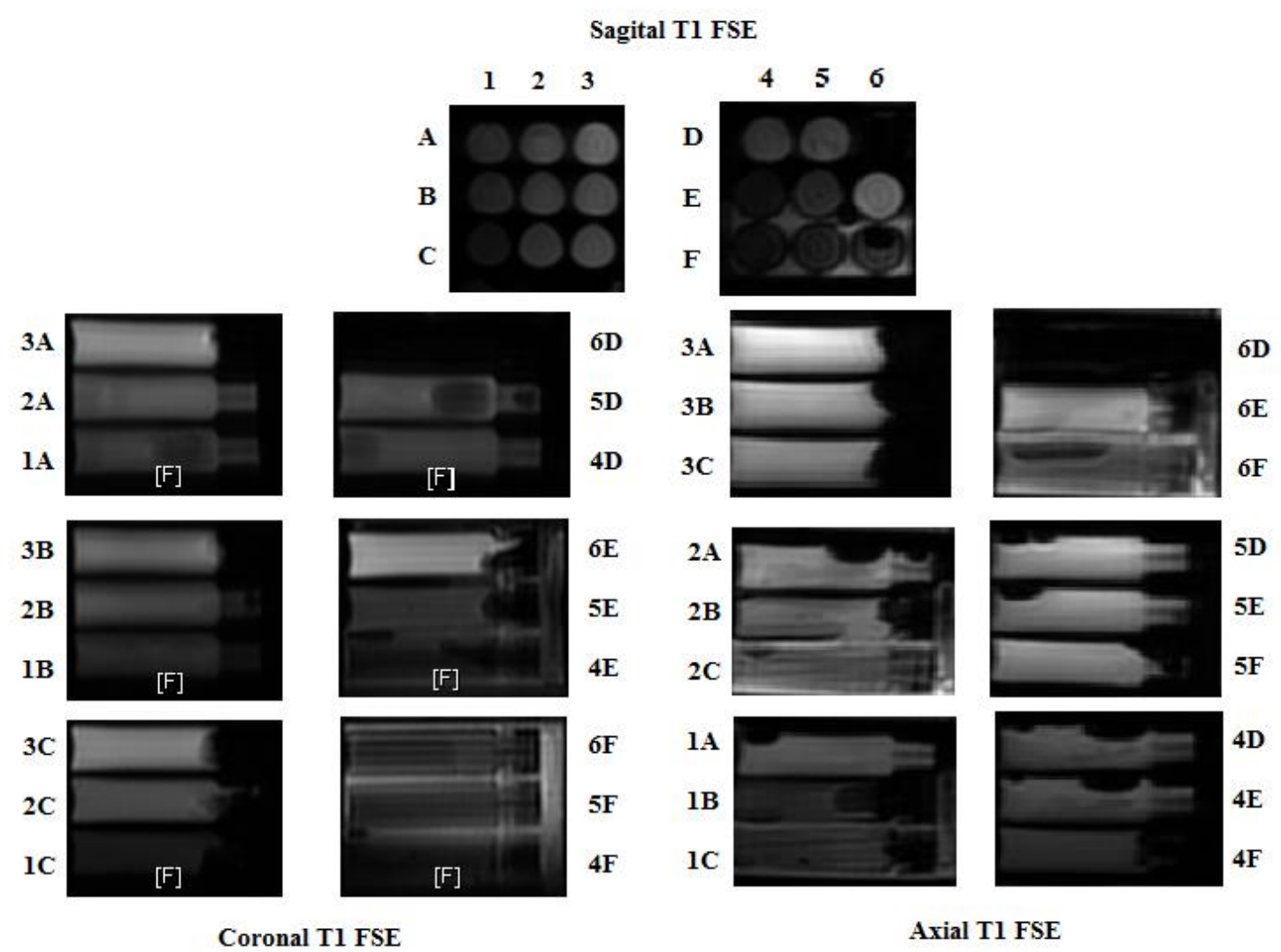

Embora as diferenças não demonstrem um comportamento semelhante entre as amostras não irradiadas e irradiadas com doses entre 10 e $50 \mathrm{kGy}$, podemos observar expressiva diferença nas tonalidades de cinza entre elas. Assim que, por exemplo, o valor de tom de cinza da amostra de gel/ $\mathrm{CuSO}_{4}(400 \mathrm{mg})$ decresce desde a amostra não irradiada, a irradiada com 10 e $50 \mathrm{kGy}$ de forma coerente. Por outro lado, as amostras de gel/CuSO $4(50 \mathrm{mg}$ e $100 \mathrm{mg})$ demonstram aumento nos valores de tons de cinza das amostras irradiadas com 10 kGy em relação às amostras irradiadas com 50 kGy. 
Tabela 1: Valores dos tons de cinza na ponderação T1 calculados pelo programa ImageJ® para amostras de compostos de gel com sulfato de cobre em diferentes proporções irradiados ou não.

\begin{tabular}{|c|c|c|c|c|c|}
\hline \multirow[b]{2}{*}{$\begin{array}{c}\text { Dose } \\
(\mathbf{k G y})\end{array}$} & \multicolumn{5}{|c|}{\begin{tabular}{|l} 
Níveis de Cinza \\
\end{tabular}} \\
\hline & $\begin{array}{c}\mathrm{Gel} / \mathrm{CuSO}_{4} \\
\quad(50 \mathrm{mg})\end{array}$ & $\begin{array}{c}\mathrm{Gel} / \mathrm{CuSO}_{4} \\
(100 \mathrm{mg})\end{array}$ & $\begin{array}{c}\mathrm{Gel} / \mathrm{CuSO}_{4} \\
(300 \mathrm{mg})\end{array}$ & $\begin{array}{c}\mathrm{Gel} / \mathrm{CuSO}_{4} \\
(400 \mathrm{mg})\end{array}$ & $\begin{array}{c}\mathrm{Gel} / \mathrm{CuSO}_{4} \\
(500 \mathrm{mg})\end{array}$ \\
\hline 0 & - & - & 93 & 63 & 35 \\
\hline 10 & 16 & 43,8 & 69 & 53 & 31 \\
\hline 50 & 32 & 94,1 & - & 43 & 56 \\
\hline
\end{tabular}

Ao contrário das amostras dos compostos, uma relação bem mais coerente entre a dose de radiação e nível de tom de cinza é possível de ser percebida nas amostras de gel puro irradiado (Fig. 3), podendo ser descrita por uma relação linear após a primeira irradiação.

Figura 3: Variação na intensidade dos tons de cinza das amostras de gel puro não irradiado e irradiados com 10, 50 e $100 k G y$.

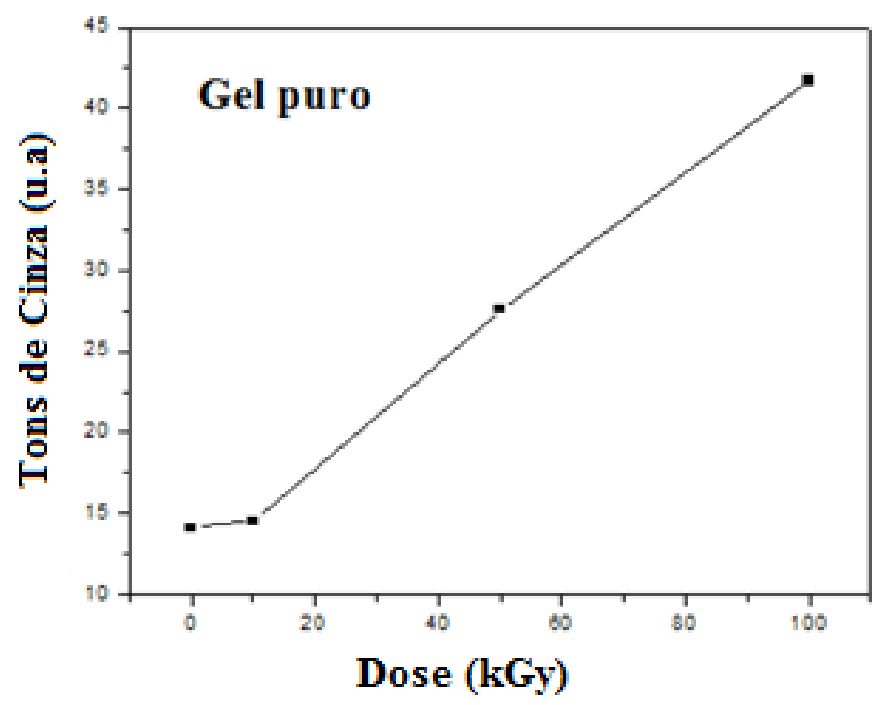

É interessante observar que a relação linear entre o nível de cinza e a dose de radiação aplicada sugere que o gel puro pode ser utilizado também para finalidade dosimétrica para estimativa de dose, muito embora a dosimetria por gel com uso de RM diagnóstica tenha sido relatada para doses inferiores, para dosimetria de tratamentos radioterápicos, por exemplo [10]. Neste contexto, abre-se 
a perspectiva para estudos futuros com irradiação em doses menores, para verificar esta potencial aplicação. Considerando ser um material barato e disponível, de densidade igual à da água, seria de grande interesse para controle volumétrico de dose, por ser um gel.

Figura 4: Imagem das amostras obtidas em ponderação T2 sequência FRFSE e a codificação das amostras conforme composição e dose de radiação recebida.

\section{SAGITAL T2 FRFSE}

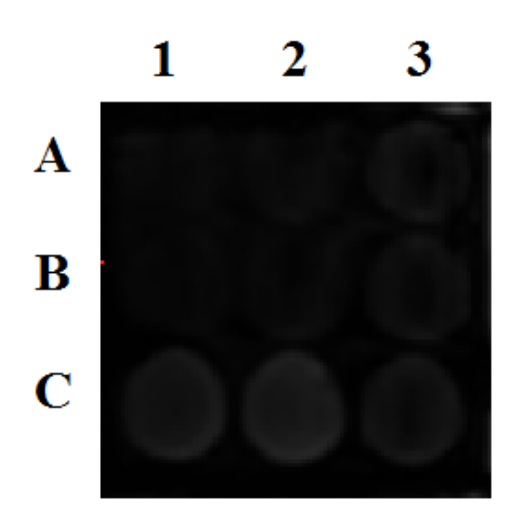

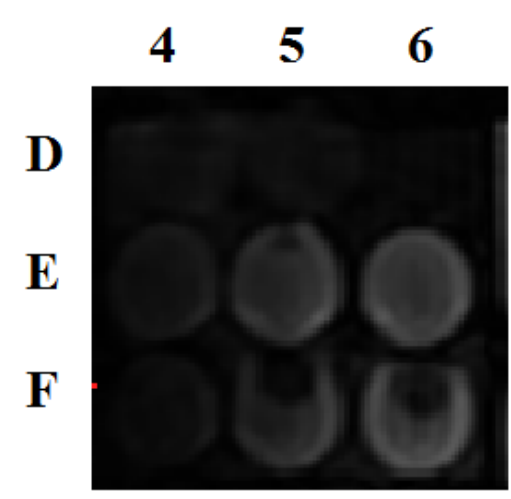

$1 \mathrm{~A}$ - Gel/CuSO4 (500 mg) não irradiado 2A - Gel/CuSO4 (400 mg) não irradiado $3 \mathrm{~A}$ - Gel/CuSO4 (300 mg) não irradiado $1 B$ - Gel/CuSO4 (500 mg) 10 kGy 2B - Gel/CuSO4 (400 mg) $10 \mathrm{kGy}$ 3B - Gel/CuSO4 (300 mg) $10 \mathrm{kGy}$ 1C - Gel/CuSO4 (50 mg) 10 kGy 2C - Gel/CuSO4 (100 mg) $10 \mathrm{kGy}$ 3C - Gel/CuSO4 (200 mg) $10 \mathrm{kGy}$ 4D - Gel/CuSO4 (400 mg) $50 \mathrm{kGy}$ $5 D$ - Gel/CuSO4 (500 mg) 50 kGy 6D - Recipiente vazio

4E - Gel puro sem irradiar $5 \mathrm{E}$ - Gel/CuSO4 (50 mg) $50 \mathrm{kGy}$ $6 \mathrm{E}-\mathrm{Gel} / \mathrm{CuSO} 4$ (100 mg) $50 \mathrm{kGy}$ 4F - Gel puro irradiado com $10 \mathrm{kGy}$ 5 F - Gel puro irradiado com $50 \mathrm{kGy}$ $6 \mathrm{~F}$ - Gel puro irradiado com $100 \mathrm{kGy}$

A Fig. 4 é analisada através da mesma metodologia aplicada para avaliação da Fig. 1 onde é possível verificar a resposta das amostras em corte sagital, agora ponderadas em T2. Observamos que na ponderação as amostras possuem um brilho reduzido em relação às ponderadas em T1. As amostras mais brilhantes são as codificadas como 1C, 2C, 5E e 6E, todas de compostos irradiados.

Através da comparação entre as Fig. 4 e Fig. 1 observamos que a amostra 4E (gel puro sem irradiar) aparece escura tanto na ponderação T1 quanto T2, demonstrando que para conseguir diferentes contrastes entre ponderações em RM é necessário a adição do sulfato de cobre ou, pelo menos, a irradiação do gel puro. Isto porque as amostras 5F e 6F (gel puro irradiadas com 50 e 100 kGy, respectivamente) aparecem escuras em T1 porém brilhantes em T2, o que também pode ser conferido na comparação apresentada na Tabela 2. Por outro lado, a amostra 4F (gel puro irradiado com $10 \mathrm{kGy}$ ) aparece escura nas duas ponderações, sugerindo a necessidade de doses acima de 50 
kGy para possibilitar a análise de diferenças de contraste entre ponderações nas amostras de gel puro.

abela 2: Valores dos tons de cinza nas ponderações T1 e T2 calculados pelo programa ImageJ® para amostras de compostos de gel puro irradiados ou não.

\begin{tabular}{ccc}
\hline & \multicolumn{2}{c}{ Níveis de Cinza } \\
\cline { 2 - 3 } $\begin{array}{c}\text { Dose } \\
(\mathbf{k G y})\end{array}$ & $\mathbf{T 1}$ & $\mathbf{T 2}$ \\
\hline 0 & 13,7 & 18,5 \\
10 & 14,5 & 12,1 \\
50 & 28,1 & 36,2 \\
100 & 45,6 & 56,5 \\
\hline
\end{tabular}

No que se refere às amostras com sulfato de cobre penta-hidratado a contribuição da irradiação se torna mais expressiva. Nota-se, por exemplo, que as amostras 1C, 2C, 5E e 6F, todas de compostos (50 e $100 \mathrm{mg}$ ) e irradiadas, são brilhantes em T2 e se apresentam obscurecidas em T1 (Tabela 1 e 3). Isto sugere que o processo de irradiação possibilita utilizar o gel com sulfato de cobre penta-hidratado para promover diferenças de resposta às ponderações, sendo mais foscas em $\mathrm{T} 1$ e brilhantes em T2, promovendo um comparativo com o contraste gordura versus água dos tecidos corporais.

Tabela 3: Valores dos tons de cinza na ponderação T2 calculados pelo programa ImageJ® para amostras de compostos de gel com sulfato de cobre em diferentes proporções irradiados ou não.

\begin{tabular}{|c|c|c|c|c|c|}
\hline \multirow[b]{2}{*}{$\begin{array}{c}\text { Dose } \\
(\mathbf{k G y})\end{array}$} & \multicolumn{5}{|c|}{ Níveis de Cinza } \\
\hline & $\begin{array}{l}{\mathrm{Gel} / \mathrm{CuSO}_{4}}^{(50 \mathrm{mg})}\end{array}$ & $\begin{array}{c}\mathrm{Gel} / \mathrm{CuSO}_{4} \\
(100 \mathrm{mg})\end{array}$ & $\begin{array}{c}\mathrm{Gel} / \mathrm{CuSO}_{4} \\
(300 \mathrm{mg})\end{array}$ & $\begin{array}{c}\mathrm{Gel} / \mathrm{CuSO}_{4} \\
(400 \mathrm{mg})\end{array}$ & $\begin{array}{c}\mathrm{Gel} / \mathrm{CuSO}_{4} \\
(500 \mathrm{mg})\end{array}$ \\
\hline 0 & - & - & 12,5 & 9,6 & 8,6 \\
\hline 10 & 19 & 24,4 & 11,5 & 8 & 6,9 \\
\hline 50 & 41,8 & 54,6 & - & 14,3 & 13,7 \\
\hline
\end{tabular}

Por outro lado, amostras em concentrações maiores de sulfato de cobre parecem não precisarem da irradiação para demonstrar diferença de contrastes em T1 e T2. Porém as amostras com concentrações acima de $300 \mathrm{mg}$ de sulfato de cobre penta-hidratado se tornam mais aquosas. Assim que para a finalidade a que se destina, ou seja, como simulador de corpo, a irradiação contribui para 
obtenção de imagens que demonstram diferentes contrastes entre as ponderações T1 e T2, mantendo a textura do gel, favorecendo seu uso seguro em exames simulados. São motivos que justificam a utilização do processo de irradiação na constituição deste instrumento didático.

\section{CONCLUSÕES}

Amostras de gel clínico incolor puro e acrescido de sulfato de cobre penta-hidratado foram testadas para obtenção de imagens em RM, com diferentes contrastes em ponderações T1 e T2 para estudo da viabilidade de seu uso como simulador de corpo. As amostras foram irradiadas com doses gama de 10, 50 e 100 kGy para promover diferença de resposta ao campo magnético. Observou-se que o processo de irradiação contribui com o contraste entre as ponderações, possibilitando associar ao comportamento da água versus gordura na composição da imagem, especialmente em amostras de gel puro ou em baixa concentração de $\mathrm{CuSO}_{4} .5 \mathrm{H}_{2} \mathrm{O}$. A adição de $\mathrm{CuSO}_{4} .5 \mathrm{H}_{2} \mathrm{O}$ em maiores proporções promove semelhante efeito, sendo então menos dependente do processo de irradiação para promoção de diferença de contraste. É interessante a opção de conseguir o mesmo efeito da adição de maiores proporções de $\mathrm{CuSO}_{4} .5 \mathrm{H}_{2} \mathrm{O}$ com a irradiação, em função da alteração da textura do gel, que se torna em alta concentração de sulfato de cobre penta-hidratado menos densa e aquosa. Isto favorece uma resposta semelhante do composto com menor quantidade de $\mathrm{CuSO}_{4} .5 \mathrm{H}_{2} \mathrm{O}$, mantendo sua textura em gel, para melhor manipulação e segurança quanto a vazamento. 


\section{AGRADECIMENTOS}

Agradecemos pelo apoio da Pró-Reitoria de Pesquisa da Universidade Federal de Minas Gerais (PRPQ - UFMG).

\section{REFERÊNCIAS}

[1] FERREIRA, F. M. Manual de Técnicas em Ressonância Magnética, $1^{\mathrm{a}}$ ed. Rio de Janeiro: Rubio, 2011.

[2] BotRanger, K. L.; LAMPignano, J. P. Manual Prático de Técnicas e Posicionamento Radiográfico, $7^{\mathrm{a}}$ ed. Elsevier, 2009.

[3] AMORIM, D. A. F. Desenvolvimento de um fantoma cerebral para ressonância magnética. Dissertação (Mestrado em Computação e Instrumentação Médica), Departamento de Física do Instituto Superior de Engenharia do Porto, Porto, 2013. http://hdl.handle.net/10400.22/7607.

[4] MITCHELl, M. D.; KUNDEL, H. L.; AXEL, L.; JOSEPH, P. M. Agarose as a tissue equivalent phantom material for NMR imaging. Magn Reson Imaging, v. 4, p. 263-266, 1986. DOI: $10.1016 / 0730-725 X(86) 91068-4$.

[5] BOLFE, V. J.; GUIRRO, R. R. J. Resistência elétrica dos géis e líquidos utilizados em eletroterapia no acoplamento eletrodo-pele. Rev Bras Fisioter, v. 13, p.499-505, 2009. http://dx.doi.org/10.1590/S1413-35552009000600006.

[6] BENZIE, R. J.; COOKE, A. H. The magnetic susceptibility of copper sulphate. Proc Phys Soc, Sect A, v. 64, p. 124-128, 1951. DOI: 10.1088/0370-1298/64/2/303.

[7] CARDOSO, G. P.; SOARES, S. S.; PEREIRA, E. L. M.; GONTIJO, R. M. G.; BATISTA, A. S. M. Radiation induction interaction between $\mathrm{CuSO}_{4}$ and clinical gel for use in MRI, In: INTERNATIONAL NUCLEAR ATLANTIC CONFERENCE, 2017, Belo Horizonte. Belo Horizonte: Comissão Nacional de Energia Nuclear, 2017. p. 1-7.

[8] CARDOSO, G. P. ; SOARES, S. S. ; GONTIJO, R.M.G. ; BATISTA, A. S. M. . Avaliação da mistura de gel clínico com sulfato de cobre como modificador dos tempos de relaxação do gel em ressonância magnética. In: CONGRESSO ANUAL DA SOCIEDADE BRASILEIRA DE 
BIOCIÊNCIAS NUCLEARES, 2017, São Paulo. Anais da Sociedade Brasileira de Biociências Nucleares. São Paulo : Sociedade Brasileira de Biociências Nucleares, 2017. p. 139. [9] DIAS, F. C. Uso do Software ImageJ para Análise Quantitativa de Imagens de Microestruturas de Materiais, Dissertação (Mestrado em Engenharia e Tecnologia Espaciais/Ciência e Tecnologia de Materiais e Sensores), Instituto de Aeronáutica e Espaço, São José dos Campos, 2008.

[10] PAVONI, J. F.; TINA, L. P.; SNOW, J.; DEWERD, L. A.; BAFFA, O. Dosimetria tridimensional usando gel MAGIC com formaldeído. Rev Bras Fis Med, v. 4(1), p. 15-18, 2010. 